\title{
Vacuum alignment and lattice artifacts
}

\author{
Maarten Golterman* \\ Department of Physics and Astronomy, San Francisco State University, \\ San Francisco, CA 94132, USA \\ E-mail: maarten@sfsu.edu
}

\section{Yigal Shamir}

Raymond and Beverly Sackler School of Physics and Astronomy, Tel Aviv University, Ramat Aviv, 69978 ISRAEL

E-mail: shamirepost.tau.ac.il

\begin{abstract}
When a subgroup of the flavor symmetry group of a gauge theory is weakly coupled to additional gauge fields, the vacuum tends to align such that the gauged subgroup is unbroken. At the same time, the lattice discretization typically breaks the flavor symmetry explicitly, and can give rise to new lattice-artifact phases with spontaneously broken symmetries. We discuss the interplay of these two phenomena, using chiral lagrangian techniques. Our first example is two-flavor Wilson QCD coupled to electromagnetism. We also consider examples of theories with staggered fermions, and demonstrate that recent claims in the literature based on the use of staggered fermions are incorrect.
\end{abstract}

The 32nd International Symposium on Lattice Field Theory

23-28 June, 2014

Columbia University New York, $N Y$

\footnotetext{
*Speaker.
} 


\section{Overview}

In two recent articles, we considered the question of what happens if, in a strongly-coupled, vector-like lattice gauge theory with a global flavor symmetry, a subgroup of this flavor symmetry is weakly gauged [1,2]. We consider the flavor gauge coupling to be "weak" if it is small at the scale of the strongly-coupled theory. In the continuum limit of such theories, the vacuum tends to align such that the weakly gauged subgroup remains unbroken [3]. However, on the lattice, even without gauging any of the flavor symmetries, it may happen that flavor symmetries are explicitly and/or spontaneously broken because of lattice artifacts. It follows that in lattice gauge theories with weakly gauged flavor symmetries, there may be a competition between vacuum alignment and lattice-induced symmetry breaking, and it is interesting to consider in more detail how this competition plays out.

Here we consider this question for two examples, the first being QCD with two flavors of Wilson fermions, and the second QCD with two flavors of (unrooted) staggered fermions. For other examples, we refer to Refs. $[1,2]$. The upshot is that indeed lattice artifacts can change the continuum phase diagram in a non-trivial way, as we will see in the examples below. We will begin with a quick review of two-flavor QCD coupled to isospin gauge fields in the continuum. We then consider lattice QCD with two flavors of Wilson fermions in Sec. 3, and with two flavors of staggered fermions in Sec. 4, after which we conclude in the final section.

\section{Continuum case}

The low-energy effective potential for two-flavor QCD with degenerate quark masses is given by

$$
\begin{aligned}
V_{\text {eff }} & =-\frac{c_{1}}{4} \operatorname{tr}\left(\Sigma+\Sigma^{\dagger}\right), \\
\Sigma & =\sigma+i \vec{\tau} \cdot \vec{\pi}, \quad \sigma^{2}+\vec{\pi}^{2}=1,
\end{aligned}
$$

in which the constant $c_{1}$ is proportional to the quark mass, and $\Sigma$ is the usual $S U(2)$-valued nonlinear pion field. Instead of using the standard exponential form for $\Sigma$, we employ the parametrization shown in Eq. (2.1), as it is more convenient in the case of $S U(2)$. We may gauge isospin (or a subgroup thereof) by considering the kinetic term of the effective lagrangian, and replacing the derivatives by covariant derivatives,

$$
\frac{f^{2}}{8} \operatorname{tr}\left(\partial_{\mu} \Sigma \partial_{\mu} \Sigma^{\dagger}\right) \rightarrow \frac{f^{2}}{8} \operatorname{tr}\left(D_{\mu} \Sigma\left(D_{\mu} \Sigma\right)^{\dagger}\right)
$$

with the covariant derivative

$$
D_{\mu} \Sigma=\partial_{\mu} \Sigma+i g\left[V_{\mu}, \Sigma\right], \quad V_{\mu}=\vec{V}_{\mu} \cdot \vec{\tau} / 2,
$$

with $V_{\mu}$ the isospin gauge field. From Eq. (2.2) one then reads off the non-derivative part

$$
\frac{g^{2} f^{2}}{4} \operatorname{tr}\left(V_{\mu}^{2}-V_{\mu} \Sigma V_{\mu} \Sigma^{\dagger}\right)
$$


and, integrating over $V_{\mu}$, this leads to an addition to the effective potential

$$
\Delta V_{\mathrm{eff}}=-\frac{g^{2} c_{3}}{8} \sum_{a} \operatorname{tr}\left(\tau_{a} \Sigma \tau_{a} \Sigma^{\dagger}\right)=-g^{2} c_{3} \sigma
$$

to lowest non-trivial order in the weak coupling $g$. We may also consider the case that we couple only a $U(1)$ subgroup of isospin $S U(2)$. Setting $V_{\mu}^{1}=V_{\mu}^{2}=0$, i.e., taking this $U(1)$ in the $\tau_{3}$ direction, one finds that

$$
\begin{aligned}
V_{\text {eff }}+\Delta V_{\text {eff }} & =-\frac{c_{1}}{4} \operatorname{tr}\left(\Sigma+\Sigma^{\dagger}\right)-\frac{e^{2} c_{3}}{8} \operatorname{tr}\left(\tau_{3} \Sigma \tau_{3} \Sigma^{\dagger}\right) \\
& =\text { constant }+\frac{1}{2} c_{1} \vec{\pi}^{2}+e^{2} c_{3} \pi^{+} \pi^{-}+\ldots
\end{aligned}
$$

We thus rederive the ancient result [4]

$$
m_{\pi^{+}}^{2}-m_{\pi^{0}}^{2}=e^{2} c_{3} / f^{2},
$$

in which we have that $c_{3}>0$ [5]. We note that in both cases gauging (a subgroup of) the isospin group stabilizes the vacuum, because $c_{3}$ is positive. This is an example of vacuum alignment [3].

\section{Wilson fermions}

In the regime in which $m / \Lambda_{\mathrm{QCD}} \sim a^{2} \Lambda_{\mathrm{QCD}}^{2}$, the leading-order effective potential for QCD with two flavors of Wilson fermion is [6]

$$
\begin{aligned}
V_{\mathrm{eff}} & =-\frac{c_{1}}{4} \operatorname{tr}\left(\Sigma+\Sigma^{\dagger}\right)+\frac{c_{2}}{16}\left(\operatorname{tr}\left(\Sigma+\Sigma^{\dagger}\right)\right)^{2} \\
& =-c_{1} \sigma+c_{2} \sigma^{2},
\end{aligned}
$$

where $c_{1} \propto m$ as before, ${ }^{1}$ and $c_{2} \propto a^{2}$, with $a$ the lattice spacing.

If $c_{2}<0$, then $\langle\sigma\rangle= \pm 1$, depending on the sign of $c_{1}$. There is a first-order phase transition at $c_{1}=0$, and isospin is always unbroken. In this case, the minimal value of the pion mass is proportional to $\left|c_{2}\right|$; no pion is ever massless as long as the lattice spacing $a>0$.

If $c_{2}>0$, one finds that the effective potential is minimized at

$$
\langle\sigma\rangle= \begin{cases}1, & c_{1} \geq 2 c_{2} \\ \frac{c_{1}}{2 c_{2}}, & -2 c_{2}<c_{1}<2 c_{2} \\ -1, & c_{1} \leq-2 c_{2}\end{cases}
$$

There is a second-order phase transition at $\left|c_{1}\right|=2 c_{2}$, and for $\left|c_{1}\right|<2 c_{2}$ we find that $|\langle\sigma\rangle|<1$, which implies that $\langle\vec{\pi}\rangle \neq 0$. $S U(2)$ isospin is spontaneously broken to a $U(1)$ subgroup, parity is spontanteously broken, and two of the three pions become massless as the Nambu-Goldstone bosons associated with this symmetry breaking. This region in the phase diagram is the Aoki phase

\footnotetext{
${ }^{1}$ The term of order $a$ in the effective potential can be, and thus has been, absorbed into $m$. For details on how this works, see Ref. [6].
} 
[7]. We note that the effect on pion masses is opposite to the effect of coupling QCD to QED, with the neutral pion being heavier than the charged pion inside the Aoki phase! ${ }^{2}$

We may now consider the combined effect of the lattice and QED, which is relevant in the case that $c_{1} \sim c_{2} \sim g^{2} c_{3}$, or $m / \Lambda_{\mathrm{QCD}} \sim a^{2} \Lambda_{\mathrm{QCD}}^{2} \sim g^{2} \sim e^{2}$. If we gauge the complete isospin group, the effective potential is

$$
V_{\text {eff }}+\Delta V_{\text {eff }}=-c_{1} \sigma+\left(c_{2}-g^{2} c_{3}\right) \sigma^{2} .
$$

The only change in comparison with Eq. (3.1) is that the low-energy constant $c_{2}$ gets replaced with $c_{2}-g^{2} c_{3}$. It follows that for $c_{2}<0$ the situation is as before, and there is a first-order transition at $c_{1}=0$. The lower bound on the pion mass gets larger by a factor $\left(\left|c_{2}\right|+g^{2} c_{3}\right) /\left|c_{2}\right|$. If $c_{2}>0$, $c_{2}-g^{2} c_{3}$ flips sign when $a \rightarrow 0$, and the Aoki phase thus gets pushed away from the continuum limit; closer to the continuum limit, when $c_{2}<g^{2} c_{3}$, the first-order scenario applies.

If we only couple electromagnetism, the effective potential is

$$
V_{\mathrm{eff}}+\Delta V_{\mathrm{eff}}=-c_{1} \sigma+c_{2} \sigma^{2}-\frac{1}{2} e^{2} c_{3}\left(\sigma^{2}+\pi_{3}^{2}\right) .
$$

This potential forces $\langle\sigma\rangle^{2}+\left\langle\pi_{3}\right\rangle^{2}=1$, and thus any Aoki condensate is now forced into the third direction. Isospin is explicitly broken, but parity remains spontaneously broken when $\left\langle\pi_{3}\right\rangle \neq 0$. There are no exact Nambu-Goldstone bosons, and inside the Aoki phase

$$
m_{\pi^{ \pm}}^{2}=e^{2} c_{3} / f^{2}, \quad m_{\pi^{0}}^{2}=2 c_{2}\left(1-\frac{c_{1}^{2}}{4 c_{2}^{2}}\right) / f^{2}
$$

Whether the charged or neutral pion mass is larger now depends on the detailed values of the various couplings.

\section{Staggered fermions}

Next, we consider QCD with two staggered fermions $\omega_{i}, i=1,2$. In the continuum limit, this theory has eight flavors, because of the four-fold doubling for each staggered fermion. ${ }^{3}$ We project these staggered fermions onto even and odd sites:

$$
\begin{array}{llrl}
\chi_{i}(x) & =\frac{1}{2}(1+\varepsilon(x)) \omega_{i}(x), & \bar{\chi}_{i}(x) & =\bar{\omega}_{i}(x) \frac{1}{2}(1-\varepsilon(x)), \\
\lambda_{i}(x) & =\frac{1}{2}(1-\varepsilon(x)) \omega_{i}(x), & \bar{\lambda}_{i}(x) & =\bar{\omega}_{i}(x) \frac{1}{2}(1+\varepsilon(x)),
\end{array}
$$

where

$$
\varepsilon(x)=(-1)^{x_{1}+x_{2}+x_{3}+x_{4}} .
$$

The massless theory with this fermion content has an exact $S U(2)_{\chi} \times S U(2)_{\lambda}$ flavor symmetry. In the continuum limit, the lattice fields $\chi_{i}$ lead to four continuum Dirac fields $\psi_{1,2,3,4}$, and likewise, the fields $\lambda_{i}$ yield four continuum Dirac fields $\psi_{5,6,7,8}[8,9]$. It is important to observe that whether

\footnotetext{
${ }^{2}$ Here the neutral pion is by definition the excitation in the direction of the condensate $\langle\vec{\pi}\rangle$, while the charged pions are the Nambu-Goldstone bosons corresponding to the broken generators.

${ }^{3}$ Often the "extra" flavors due to this doubling are referred to as "taste" instead of flavor, but here we will refer to all fermions as flavors in the continuum limit.
} 
any of the exact lattice symmetries are to be considered as axial symmetries depends on what type of mass term is added to the theory. Here we will keep the theory massless, but instead consider the effect of weakly gauging (some of) the exact flavor symmetries.

On the lattice, dynamical symmetry breaking is expected to take place, and various condensates may form as a consequence. One possible condensate is $\sum_{k=1}^{8} \bar{\psi}_{k} \psi_{k}$, which is the continuum limit of

$$
\sum_{i, \mu}\left(\bar{\chi}_{i}(x) U_{\mu}(x) \chi_{i}(x+\mu)+\bar{\lambda}_{i}(x) U_{\mu}(x) \lambda_{i}(x+\mu)+\text { h.c. }\right) .
$$

This operator is invariant under $S U(2)_{\chi} \times S U(2)_{\lambda}$; note that an operator such as $\bar{\chi}_{i}(x) \chi_{i}(x)$ cannot be constructed because of the projections in Eq. (4.1). The minimum number of links in any lattice operator with continuum limit $\sum_{k=1}^{8} \bar{\psi}_{k} \psi_{k}$ is one. A different condensate, corresponding to a singlesite operator

$$
\sum_{i}\left(\bar{\chi}_{i}(x) \lambda_{i}(x)+\bar{\lambda}_{i}(x) \chi_{i}(x)\right)
$$

also exists; in the continuum limit it takes the form

$$
\bar{\psi}_{5} \psi_{1}+\bar{\psi}_{6} \psi_{2}+\bar{\psi}_{7} \psi_{3}+\bar{\psi}_{8} \psi_{4}+\text { h.c. . }
$$

In contrast to the one-link operator (4.3), this latter condensate breaks $S U(2)_{\chi} \times S U(2)_{\lambda}$ down to $S U(2)_{\text {diag. }}$. Clearly, these two condensates are not equivalent on the lattice, even if they are in the continuum. In the continuum, the flavor group enlarges to $S U(8) \times S U(8)$, both condensates break this to $S U(8)_{\text {diag }}$, and they can be rotated into each other by the continuum flavor group. All we need to support this observation is to assume that the continuum limit of the lattice theory is universal.

The two condensates are no longer equivalent when we gauge some subgroup of the lattice symmetry group, even in the continuum limit, as we will show through two examples. First, let us gauge just the $U(1)$ group of transformations generated by $T_{3}^{\varepsilon}$, where

$$
T_{3}^{\varepsilon}=T_{3}^{\chi}-T_{3}^{\lambda}
$$

Here $T_{a}^{\chi}$ and $T_{a}^{\lambda}$ are the generators of $S U(2)_{\chi}$ and $S U(2)_{\lambda}$, respectively. To leading order in the weak gauge coupling $e$, the effective potential generated by the weak gauge fields is [3]

$$
V_{\mathrm{eff}}=-e^{2} C \operatorname{tr}\left(\Sigma Q_{R} \Sigma^{\dagger} Q_{L}\right)
$$

with $\Sigma \in S U(8)$ the non-linear Nambu-Goldstone field, $C>0$ [5], and in which $Q_{R}$ and $Q_{L}$ are spurion fields. In order to represent the coupling to the $U(1)$ gauge field they need to be fixed; to what values depends on the basis, as we will see below. Once we choose $Q_{R}$ and $Q_{L}$, this potential breaks $S U(8) \times S U(8)$, and the one-link and single-site condensates are no longer degenerate. Let us work out in more detail what this implies for the condensate $\langle\Sigma\rangle$.

On the one-link basis, i.e., the basis on which the one-link condensate is diagonal in the continuum limit, the corresponding lattice one-link mass term (4.3) does not break the $U(1)$ symmetry. On this basis, the generator for $U(1)$ transformations takes the form [2] ${ }^{4}$

$$
T_{3}^{\varepsilon}=\tau_{3} \times \tau_{3} \times I_{2}
$$

\footnotetext{
${ }^{4} I_{n}$ is the $n \times n$ identity matrix.
} 
This indeed looks vector-like, and we thus have that

$$
Q_{R}=Q_{L}=T_{3}^{\varepsilon} \quad \rightarrow \quad V_{\text {eff }}=-e^{2} C \operatorname{tr}\left(\Sigma T_{3}^{\varepsilon} \Sigma^{\dagger} T_{3}^{\varepsilon}\right),
$$

with values $V_{\text {eff }}\left(\Sigma_{1-\text { link }}\right)=-24 e^{2} C$ and $V_{\text {eff }}\left(\Sigma_{\text {site }}\right)=+24 e^{2} C$. Here $\Sigma_{1-\text { link }}=I_{8}$ corresponds to the continuum condensate $\sum_{k=1}^{8} \bar{\psi}_{k} \psi_{k}$, with lattice version (4.3), and $\Sigma_{\text {site }}=\tau_{1} \times I_{4}$ corresponds to the continuum condensate (4.5), with lattice version (4.4).

It is instructive to check this result on a different basis, the single-site basis on which the single-site condensate is, by definition, diagonal in the continuum limit. ${ }^{5}$ The basis transformation taking the one-link basis into the single-site basis takes $T_{3}^{\varepsilon}$ into [2]

$$
\tilde{T}_{3}^{\varepsilon}=-\gamma_{5}\left(\tau_{2} \times I_{2} \times I_{2}\right),
$$

where we use tildes to indicate that we are now working on the single-site basis. This has the appearance of an axial symmetry; and we have that

$$
Q_{R}=-Q_{L}=\tilde{T}_{3}^{\varepsilon} \quad \rightarrow \quad V_{\mathrm{eff}}=+e^{2} C \operatorname{tr}\left(\tilde{\Sigma} \tilde{T}_{3}^{\varepsilon} \tilde{\Sigma}^{\dagger} \tilde{T}_{3}^{\varepsilon}\right)
$$

where in the effective theory the $\gamma_{5}$ is omitted from $\tilde{T}_{3}^{\varepsilon}$. The minus sign between $Q_{R}$ and $Q_{L}$ follows from the presense of the $\gamma_{5}$ in Eq. (4.10). On this basis, on which by definition the singlesite condensate takes the form $\tilde{\Sigma}=I_{8}$, it is straightforward to show that the one-link condensate has flavor structure $\tilde{\Sigma}=\tau_{3} \times I_{4}$, and the effective potential (4.7) takes the values $V_{\text {eff }}\left(\tilde{\Sigma}_{1-\text { link }}=\right.$ $\left.\tau_{3} \times I_{4}\right)=-24 e^{2} C$ and $V_{\text {eff }}\left(\tilde{\Sigma}_{\text {site }}=I_{8}\right)=+24 e^{2} C$. Never mind what basis we use to find the pattern of symmetry breaking, the result is that the condensate minimizing $V_{\text {eff }}$ is the one-link condensate, which leaves the $U(1)$ unbroken.

As in the previous section, we also consider the example in which we introduce weakly coupled gauge fields for the whole group $S U(2)_{\chi} \times S U(2)_{\lambda}$. An intriguing possibility would be that the condensate breaks this group down to a smaller group, producing a dynamical Higgs mechanism that would render some of the weak gauge boson massive. Now the effective potential generated by the weak gauge fields is

$$
V_{\mathrm{eff}}=-g_{\chi}^{2} C \sum_{a} \operatorname{tr}\left(\Sigma T_{a}^{\chi} \Sigma^{\dagger} T_{a}^{\chi}\right)-g_{\lambda}^{2} C \sum_{a} \operatorname{tr}\left(\Sigma T_{a}^{\lambda} \Sigma^{\dagger} T_{a}^{\lambda}\right)
$$

The low-energy constant $C$ is the same as in Eq. (4.7). Working on the one-link basis, we find that $V_{\text {eff }}\left(\Sigma=I_{8}\right)=-12\left(g_{\chi}^{2}+g_{\lambda}^{2}\right) C$, while $V_{\text {eff }}\left(\Sigma=\tau_{1} \times I_{4}\right)=0$, and again the one-link condensate $\Sigma=I_{8}$ is the absolute minimum. Again, the vacuum alignment mechanism causes the flavor group $S U(2)_{\chi} \times S U(2)_{\lambda}$ to remain unbroken, and no dynamical Higgs mechanism takes place. As before, this exercise can be repeated on the single-site basis, with, of course, the same conclusion.

\section{Conclusion}

We have shown, through examples, how lattice artifacts, quark-mass induced contributions, and weak interactions can all compete in determining the pattern of symmetry breaking in a strongly

\footnotetext{
${ }^{5}$ For the explicit construction of this basis, see Ref. [2].
} 
coupled gauge theory. Of course, in the continuum limit, quark-mass and weak-coupling effects dominate, but these examples demonstrate that lattice artifacts can mask the correct phase diagram of the theory. We find that the mechanism of vacuum alignment prevents a condensate that would imply a dynamical Higgs mechanism in the weak sector from developing. This disproves claims to the contrary in the literature [10]. The only assumption underlying our conclusions is that of universality. In the staggered case, universality implies the equivalency of the single-site and onelink condensates in the continuum limit, in the absence of the coupling to weak gauge fields. Close enough to the continuum limit, only the weak interactions break the global flavor symmetry, and thus only weak interactions determine the phase diagram.

This type of analysis can be extended to composite Higgs models of interest to BSM physics. In Ref. [1] we considered for instance the $S U(5) / S O(5)$ coset model of relevance for the "Littlest Higgs" model [11]. In Ref. [2] we also consider a staggered example with only six flavors in the continuum limit, by making use of one normal and one reduced staggered fermion $[12,8,9]$. As a corrolary of our discussion, we observe that in order to determine the low-energy constants $c_{3}$ in the Wilson case, and $C$ in the staggered case, weak gauge fields only need to be coupled to flavor symmetries that are exact on the lattice. This observation generalizes to other cases of interest (such as the $S U(5) / S O(5)$ coset model) as well.

\section{Acknowledgements}

We acknowledge discussions with Simon Catterall. MG thanks the School of Physics and Astronomy of Tel Aviv University and YS thanks the Department of Physics and Astronomy of San Francisco State University for hospitality. MG is supported in part by the US Department of Energy, and YS is supported by the Israel Science Foundation under grants no. 423/09 and 449/13.

\section{References}

[1] M. Golterman and Y. Shamir, Phys. Rev. D 89, 054501 (2014) [arXiv:1401.0356 [hep-lat]].

[2] M. Golterman and Y. Shamir, Phys. Rev. D 89, 074502 (2014) [arXiv:1401.3151 [hep-lat]].

[3] M. E. Peskin, Nucl. Phys. B 175, 197 (1980).

[4] T. Das, G. S. Guralnik, V. S. Mathur, F. E. Low, J. E. Young, Phys. Rev. Lett. 18, 759 (1967).

[5] E. Witten, Phys. Rev. Lett. 51, 2351 (1983).

[6] S. R. Sharpe and R. L. Singleton Jr., Phys. Rev. D 58, 074501 (1998) [arXiv:hep-lat/9804028].

[7] S. Aoki, Phys. Rev. D 30, 2653 (1984).

[8] C. van den Doel and J. Smit, Nucl. Phys. B 228, 122 (1983).

[9] M. Golterman and J. Smit, Nucl. Phys. B 245, 61 (1984).

[10] S. Catterall and A. Veernala, Phys. Rev. D 88, 114510 (2013) [arXiv:1306.5668 [hep-lat]]; arXiv:1401.0457 [hep-lat].

[11] N. Arkani-Hamed, A. G. Cohen, E. Katz and A. E. Nelson, JHEP 0207 (2002) 034 [hep-ph/0206021].

[12] H. S. Sharatchandra, H. J. Thun and P. Weisz, Nucl. Phys. B 192, 205 (1981). 